\title{
Use of internet data to track Chinese behavior and interest in COVID-19
}

\author{
Tengteng Wang ${ }^{1}$, Xiude Chen ${ }^{1}$, Qi Zhang ${ }^{1}$, Xunbo Jin ${ }^{1 *}$ \\ ${ }^{1}$ Department of Urology, Shandong Provincial Hospital Affiliated to Shandong University, \\ Jinan, Shandong, 250021, China \\ *Corresponding Author: \\ Xunbo Jin, M.D, Ph.D \\ Department of Urology, Shandong Provincial Hospital Affiliated to Shandong University, \\ Jinan, Shandong, 250021, China \\ Tel: +8613573109063
}

mail:357006250@qq.com

\begin{abstract}
Background: During the novel coronavirus pneumonia (COVID-19) outbreak, the internet became the main channel for the public to access all kinds of information. Through search engines and other media, people can acquire information about the epidemic situation in real time. Baidu, currently the top online search tool in China, has developed an internet search trends gathering and analysis tool called Baidu Index.

Purpose: To understand the public's focus on the epidemic and their most pressing needs through analysis of Chinese internet usage during the first month of the outbreak, which was also the most severe period.
\end{abstract}


Method: The data period selected in this study was from January 23, 2020 to February 22, 2020. With the help of Baidu index, we identified popular search terms related to the novel coronavirus which were grouped into categories; "basic information of the disease", "latest developments of epidemic", "diagnosis and treatment of the novel coronavirus pneumonia" and "extended information". Trend analysis was obtained depicting the quantities with which each category was searched over the study period. Next, we selected the category with the highest search volume, and obtained data regarding geographic and demographic characteristics nationally. Finally, we focused on Wuhan, the most affected city, and explored the local residents' interest in COVID-19 by tracking their online seeking behavior and analyzing their most searched terms.

Results: The general public was most concerned about news of "latest developments of epidemic", which maintained a high level of attention throughout the study period. The remaining categories in descending order of attention received were "basic information of disease", "extended information" and "diagnosis and treatment of the novel coronavirus pneumonia". As time went by, search index of these categories showed a significant decline after the early rapid growth. The mobile search volume was much higher than the PC search volume. Most of the people searching for information about the "latest developments of epidemic" came from economically developed provinces and cities with a large floating population. The group with the maximum concern were young and middle-aged men aged 20 49. The most searched terms by Wuhan residents were "how to use a thermometer", "n95 mask", "testing kit" and "nucleic acid testing".

Conclusion: Through big data from internet, we are able to accurately understand what people are concerned about and what they need during the epidemic. If this data is used 
in media coverage, public health policy decision-making, personal epidemic prevention education, and so on, it will certainly play an important role. Other countries and regions in the early stages of the epidemic can also benefit from China's experience.

\section{Keywords}

internet data, Baidu Index, COVID-19, novel coronavirus pneumonia

\section{Introduction}

Since January 2020, the novel coronavirus pneumonia spread rapidly in China and the world, and became a Public Health Emergency of International Concern (PHEIC). It has caused great harm to people's health and safety worldwide ${ }^{1,2}$. Unlike the SARS outbreak in 2003, during the COVID-19 outbreak the internet has become the main source for individuals to access all kinds of information. Through search engines, social media, news clients and other media, people can access the epidemic information in real time $e^{3,4}$. According to the latest China Statistical Report on Internet Development, there are 854 million internet users in the country, $81.3 \%$ of whom reported to use search engines to obtain information $^{3}$. After withdrawal of Google from China in 2010, Baidu is now China's number one search engine, with about $90.9 \%$ of internet users ranking Baidu as its preferred and dominant search engine ${ }^{5}$. It has become a habit and custom to use Baidu to obtain all kinds of information including those related to diseases. Since the outbreak

of the COVID-19 epidemic, internet users have searched and browsed information about the new coronavirus through Baidu more than 1 billion times per day, revealing a strong willingness to actively obtain information. When people search and browse the internet, large data will be generated automatically, through tracking and recording of search 
histories. In order to analyze these data, Baidu has developed Baidu Index, a tool for the purpose of gathering online strategies and internet search trend, which is in turn used to capture public interest and demand ${ }^{6}$. Our study used Baidu Index to analyze a vast amount of data on information regarding the epidemic obtained by the Chinese people through the internet during the most severe period of the outbreak, the first month, in order to understand the public concerns and their most pressing needs. This information will help us to provide more targeted informatics services for the general public and provide a scientific basis for formulating reasonable epidemic prevention and control measures.

\section{Method}

1. Overview of the study tool: Baidu Index

Baidu Index is a big data sharing platform composed of vast amounts of information generated by the search behaviors of those using the Baidu search engine. It reflects the active search demand of netizens, and all activities affecting their online search behavior may affect Baidu Index. The data is updated daily in real time, collecting search information from all PCs since June 2006, and all mobile devices since January 2011. Each search query returns a trend analysis diagram, a geographic and demographic distribution, and other representations of the accumulated data ${ }^{7}$.

The trend analysis is based on the search index of the included study subjects. Trend can be analyzed over different time periods or within different geographical regions. The overall data, PC data and mobile data can be provided separately. The relevant keywords searched via the Baidu search engine are the points of statistical interest. The weighted sum of the search frequencies of the popular keywords can be utilized to rank the 
predetermined corresponding broader categories by search volume. The geographic analysis quantitates the distribution of search volumes of interest across province, city and so on. The demographic analysis quantitates the distribution of search volumes of interest across various age groups and sex.

\section{Searching Strategy}

Period of data acquisition in this study ranged from January 23, 2020 to February 22, 2020, which was the worst month of the epidemic in China. On January 23, Wuhan City was locked down, and on January 24, several provinces or municipalities directly under the Central Government in China launched a first-class response to major public health emergencies. On February 22, patients diagnosed across the country reached a peak. With the help of Baidu index, we grouped popular search terms related to the novel coronavirus into categories as outlined in Table 1, which consist of "basic information of disease", "latest development of epidemic", "diagnosis and treatment of the new coronavirus pneumonia" and "extended information" and then carried out the search trend study, respectively. Furthermore, we selected the category with the highest search volume, and obtained data related to geographic and demographic distribution. Finally, we focused on Wuhan, the city most affected by the epidemic, and investigated the information local residents were most concerned about by tracking their online seeking behavior.

\section{Results}

The general public was most concerned about information relevant to the "latest developments of epidemic" category, which maintained a high level of attention throughout the study period. The remaining categories, arranged in descending order of 
attention received, were "basic information of disease", "extended information" and "diagnosis and treatment of the novel coronavirus pneumonia" (Table 1), the search volume of which showed a significant decline over time after an early surge (Figure 1). After exploring the different sources of information, we found that the mobile search volume is much higher than the PC search volume (Figure 2).

The geographic distribution of targeted population was represented in the map of China generated by the Baidu index. Overall, the search for terms relevant to "latest developments of epidemic" came mostly from economically developed provinces and cities with large mobile populations (Figure 3). The age distribution indicated that the group responsible for the most searches were age 20 29 years, followed by 30 39 and 40 49 years age group. These age groups count for more than $80 \%$ of the user population. In terms of gender distribution, the number of males who searched the analyzed terms was significantly greater than that of females (Figure 4). This suggested that young and middle-aged men were the most likely in the population to have acquired information regarding the novel coronavirus pneumonia through the internet.

In Wuhan, where the epidemic was most serious, after the city was locked down, the most searched keywords in the city were related to self-protection and included "how to use a thermometer" and "n95 mask". Additional foci of local user's attention were "testing kit" and "nucleic acid testing". Thus overall, the frequently searched keywords are related to disease prevention and diagnosis, verifying short supplies of medical resources in Wuhan, especially the shortage of testing kits (Figure 5).

\section{Discussion}

Presently, we are in the mobile era of the internet, and mobile internet has become the 
infrastructure of big data. Unlike traditional internet accessed through PCs, mobile internet exhibits a closer link with people's daily activities. People use mobile phones all the time, which generates various behavioral data. Additionally, various mobile phone apps and operators have user identification methods that can link data with corresponding users $^{8}$. These factors have resulted in an explosive growth of information, which makes the original basic data management method of relying solely on the collection, storage and query of data no longer practical. Therefore, there is an urgent need for effective data processing and analysis to extract valuable information from the growing expanse of data $^{9}$. In China, internet technology services and applications are playing an increasingly important role in combating new coronavirus infections. Baidu, for example, has become the most popular portal for people to access authoritative information and knowledge in this epidemic. Using Baidu Index, a large data gathering and processing tool, we have opportunities to understand the most real and urgent demands of the general public accurately and globally in the event of an outbreak of COVID-19.

In this study, we found that the public's concerns about the novel coronavirus pneumonia were both regarding prevention and remaining informed. Among the predetermined categories, information relevant to the "latest developments of epidemic" category was the most searched, and had maintained a high degree of attention throughout the study period. After rapid growth in the early stage, the search volume for "basic information of disease", "extended information" and "diagnosis and treatment of the novel coronvairus pneumonia" showed a significant decline over time. This may be related to the urgency to obtain general disease relevant knowledge at the early stage of a sudden outbreak, when the public's knowledge, recognition and protection measures against the virus is almost 
zero $^{10,11}$. However, with the timely and transparent release of all kinds of authoritative information by the government and the media, and sufficient supply of epidemic prevention materials, the public's sense of security gradually improved, and the collective panic had been significantly eased ${ }^{12,13}$. Previous studies have shown that there was a link between the number of media reports and the number of infections transmitted. When the media coverage increased tenfold, the number of infections of such diseases decreased by $33.5 \%{ }^{14}$. The reason is that the spread of disease occurs in dynamic social activities, so individual actions have an important impact on the spread. The higher the media coverage, the better the public awareness of prevention, and the slower the spread of disease. Therefore, timely, transparent and authoritative information disclosed by the media may be an effective means of preventing the spread of disease ${ }^{15,16}$. Such a purpose has been one of the largest utilizations of the internet during the coronavirus epidemic. Based on the needs of the public, the network media launched the novel coronavirus pneumonia channel, which presents a geographic map of infections, the latest developments, epidemic prevention knowledge and other multi-dimensional information ${ }^{17,18}$. In addition, Baidu Map first launched a "fever clinic map" in the four cities of Beijing, Wuhan, Shanghai and Chengdu. If one feels sick, he or she can quickly find the nearest fever clinic through the map for medical treatment ${ }^{17}$. The e-commerce platform has also introduced a number of measures to monitor the sources and ensure the steady supply of protective materials such as masks, in turn promoting supply market stability ${ }^{13}$. These big data-based measures have played a positive role in the prevention and control of the epidemic ${ }^{19}$. Through analysis of the source of data origination, we found that the absolute majority of the population used mobile devices to search for 
information, which may be attributed to the popularity of domestic smart phones, making it convenient for people to access a variety of information through the mobile internet. With this finding, policy makers and information publishers can take advantage of tools such as mobile social media and mobile news clients to better access the targeted groups. According to the geographical distribution map, most of the people searching for terms within the "latest developments of epidemic" category were from the provinces and cities with developed economies and large floating populations. People in these regions were more concerned about the epidemic because of several reasons: these regions had relatively more severe disease status; they had better internet infrastructure; they had a large residential population with a higher proportion of those who had a stronger awareness of health issues. In terms of population characteristics, the $20 \sim 49$ years age group showed the highest volume of searches, among whom the number of men were significantly greater than women. Although a high-risk group of this novel coronavirus infection, middle aged and older people committed relatively less attention to the disease on the internet ${ }^{1}$. This suggested that this latter group should be addressed through the development of unique educational and prevention material via methods that they were more familiar with and receptive to.

Finally, in our search data analysis, we focused on Wuhan, the worst-hit city of the epidemic. Unlike the rest of the country, Wuhan residents were most concerned about "how to use a thermometer", "n95 mask", "testing kit" and "nucleic acid testing". These searched keywords are related to disease prevention and diagnosis. The continuous high level of attention verified the urgent situation of the medical resources in Wuhan. Based on this result, the government can make data driven decisions, to carry out education 
regarding prevention, such as how to correctly select and use masks, safe use of disinfectant, and how to correctly monitor body temperature etc. At the same time, the data can be utilized in the informed dispatch of the medical protection material. Through big data analysis, predictions can be made to identify the most urgent epidemic areas, in consideration with delivery times and logistics, to triage and efficiently distribute materials directly from the production lines. Such a method of identifying regional needs and providing expedited assistance, would serve to comfort the general public and avoid the epidemic causing more serious chain reactions.

When a major event occurs, searching the internet is the preferred medium to obtain information and knowledge ${ }^{20}$. The allure is real-time news delivery, as the public demands up to date information and knowledge ${ }^{21}$. The con is that the internet is additionally flooded with inaccurate information, which may serve to fuel worries and incite public panic ${ }^{22}$. However completely blocking the wealth of information the internet provides, would cause even bigger panic, and result in a fertile ground for unchecked rumors $^{23}$. For this reason, the major internet services/applications have opened rumorrefuting platforms which aim to rapidly dispel rumors and false information related to the epidemic, through the analysis and sharing of large amounts of data. Ultimately these measures effectively maintain the sanctity of accurate information dissemination and help netizens to have a clear understanding of the epidemic situation as well as guidance on how to proceed $^{24}$.

\section{Conclusion}

With the help of big data from the internet, we can accurately and globally understand what people care about and what their pressing needs are during the epidemic. This 
information can play a vital role and be utilized in media coverage, health policy decision-making, personal epidemic prevention education, and so on. Other countries and regions in the early stages of the epidemic can also benefit from China's experience.

\section{Conflicts of Interest}

None declared.

\section{Abbreviations}

COVID-19 Coronavirus Disease 19

SARS Severe Acute Respiratory Syndrome

PC Personal Computer

\section{Reference}

1. Wu Z, McGoogan JM. Characteristics of and Important Lessons From the Coronavirus Disease 2019 (COVID-19) Outbreak in China: Summary of a Report of 72314 Cases From the Chinese Center for Disease Control and Prevention. Jama. 2020.

2. Sohrabi C, Alsafi Z, O'Neill N, et al. World Health Organization declares global emergency: A review of the 2019 novel coronavirus (COVID-19). Int J Surg. 2020;76:71-76.

3. CINIC. ( 2019 ) the 44th China Statistical Report on Internet Development. Available from:http://www.cnnic.net.cn/hlwfzyi/hlwxzbg/hlwtibg/201908/P020190830356787490958.pdf.

4. Hernández-García I, Giménez-Júlvez T. Assessment of Health Information About COVID-19 Prevention on the Internet: Infodemiological Study. JMIR Public Health Surveill. 2020;6(2):e18717.

5. CINIC ( 2019 ) China Statistical Report on Search Engine.Available from: http://www.cnnic.net.cn/hlwfzyi/hlwxzbg/ssbg/201910/P020191025506904765613.pdf.

6. Vaughan LaC, Y. Data Mining From Web Search Queries: A Comparison of Google Trends and Baidu Index. 2015 May 19;66(1):13-22.

7. Baidu Index. (2020). Available from http://index.baidu.com/.

8. Chen M, Mao S, Liu Y. Big Data: A Survey. Mobile Networks and Applications. 2014 April 1;19(2):171-209.

9. Eysenbach G. Infodemiology and infoveillance: framework for an emerging set of public health informatics methods to analyze search, communication and publication behavior on the Internet. J Med Internet Res. 2009;11(1):e11.

10. Deng SQ, Peng HJ. Characteristics of and Public Health Responses to the Coronavirus Disease 2019 Outbreak in China. J Clin Med. 2020;9(2).

11. Leung CC, Lam TH, Cheng KK. Mass masking in the COVID-19 epidemic: people need guidance. Lancet. 2020;395(10228):945.

12. National Health Commission of the People's Republic of China. Notice on accomplishing online consultation services in the epidemic prevention and control (Medical Letter [2020] No.112). National Heallth Commission of the People's Republic of China; 2020-02-07; Available from:http://www.nhc.gov.cn/yzygi/s7652m/202002/32c3e98988894fa18280e4543d2710c7.shtm l.

13. The State Council's Comprehensive Team of China for Joint Prevention and Control of New Coronavirus Pneumonia. Introduction of the latest progress in the production, dispatching and 
import of epidemic prevention materials. The State Council's Comprehensive Team for Joint Prevention and Control of New Coronavirus Pneumonia; 2020-2-5; Available from:http://www.gov.cn/xinwen/gwylflkjz/index.htm.

14. Kim L, Fast SM, Markuzon N. Incorporating media data into a model of infectious disease transmission. PLoS One. 2019;14(2):e0197646.

15. Lin L, Jung M, McCloud RF, Viswanath K. Media use and communication inequalities in a public health emergency: a case study of 2009-2010 pandemic influenza A virus subtype H1N1. Public Health Rep. 2014;129 Suppl 4(Suppl 4):49-60.

16. Gu H, Chen B, Zhu H, et al. Importance of Internet surveillance in public health emergency control and prevention: evidence from a digital epidemiologic study during avian influenza A H7N9 outbreaks. J Med Internet Res. 2014;16(1):e20.

17. Chen S, Yang J, Yang W, Wang C, Bärnighausen T. COVID-19 control in China during mass population movements at New Year. Lancet. 2020;395(10226):764-766.

18. Liu S, Yang L, Zhang C, et al. Online mental health services in China during the COVID-19 outbreak. Lancet Psychiatry. 2020;7(4):e17-e18.

19. Xiaofeng. JXZ. Emergency Intelligence Service of Think Tanks Based on the Construction of Government Big Data Capability : Taking Prevention of COVID-19 for Example. . LIBRARY\&INFORMATION. 2020 Feb 20;(01):64-74.

20. Tang L, Bie B, Park SE, Zhi D. Social media and outbreaks of emerging infectious diseases: A systematic review of literature. Am J Infect Control. 2018;46(9):962-972.

21. Jernigan DB. Update: Public Health Response to the Coronavirus Disease 2019 Outbreak - United States, February 24, 2020. MMWR Morb Mortal Wkly Rep. 2020;69(8):216-219.

22. Mian A, Khan S. Coronavirus: the spread of misinformation. BMC Med. 2020;18(1):89.

23. Ioannidis JPA. Coronavirus disease 2019: the harms of exaggerated information and nonevidence-based measures. Eur J Clin Invest. 2020:e13223.

24. Depoux A, Martin S, Karafillakis E, Bsd RP, Wilder-Smith A, Larson H. The pandemic of social media panic travels faster than the COVID-19 outbreak. J Travel Med. 2020. 
Table 1. Hot search terms related to COVID-19

\begin{tabular}{|c|c|c|}
\hline Rank & Category & Search Terms (Daily average search index/mobile index) \\
\hline 1 & $\begin{array}{l}\text { Latest development of } \\
\text { epidemic }\end{array}$ & $\begin{array}{l}\text { Real-time status of novel pneumonia }(3680044 / 3507023) \\
\text { Latest news about coronavirus }(87854 / 86286) \\
\text { Latest news about Pneumonia }(154908 / 148312) \\
\text { Epidemic map }(125205 / 110613)\end{array}$ \\
\hline 2 & $\begin{array}{l}\text { Basic information of } \\
\text { disease }\end{array}$ & $\begin{array}{l}\text { Novel coronavirus }(1674055 / 1583386) \\
\text { Novel coronavirus pneumonia }(542856 / 437603) \\
\text { Features of novel coronavirus }(372467 / 264048) \\
\text { Pneumonia }(344078 / 321595) \\
\text { Images of coronavirus }(4983 / 4444)\end{array}$ \\
\hline 3 & Extended information & $\begin{array}{l}\text { SARS }(660320 / 632984) \\
\text { N95 mask }(271350 / 258467) \\
\text { Mask }(63251 / 53800) \\
\text { Surgical mask }(6396 / 5632) \\
\text { Fever clinic }(1854 / 1334) \\
\text { Mask machine }(1723 / 1350)\end{array}$ \\
\hline 4 & Symptoms and treatments & $\begin{array}{l}\text { Symptoms of COVID-19 }(259435 / 250556) \\
\text { What are the symptoms for pneumonia }(133046 / 130403) \\
\text { How to prevent the novel coronavirus }(14297 / 13210) \\
\text { Dry cough }(10009 / 9255) \\
\text { Cough }(8538 / 8385) \\
\text { How to treat coronavirus }(3585 / 3277) \\
\text { Fever }(3267 / 2788) \\
\text { Pneumonia vaccine }(2441 / 2080) \\
\text { Coronavirus vaccine }(1334 / 1107)\end{array}$ \\
\hline
\end{tabular}

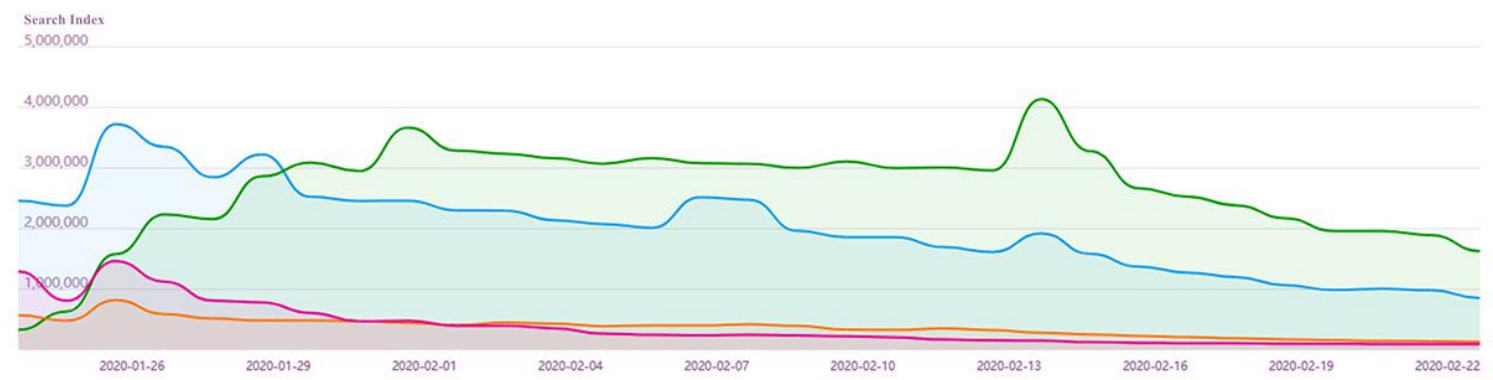

Figure 1. Search trends of hot search terms related to COVID-19 (green line: latest development of epidemic, blue line: basic information of disease, red line: extended information, orange line: symptoms and treatments) 


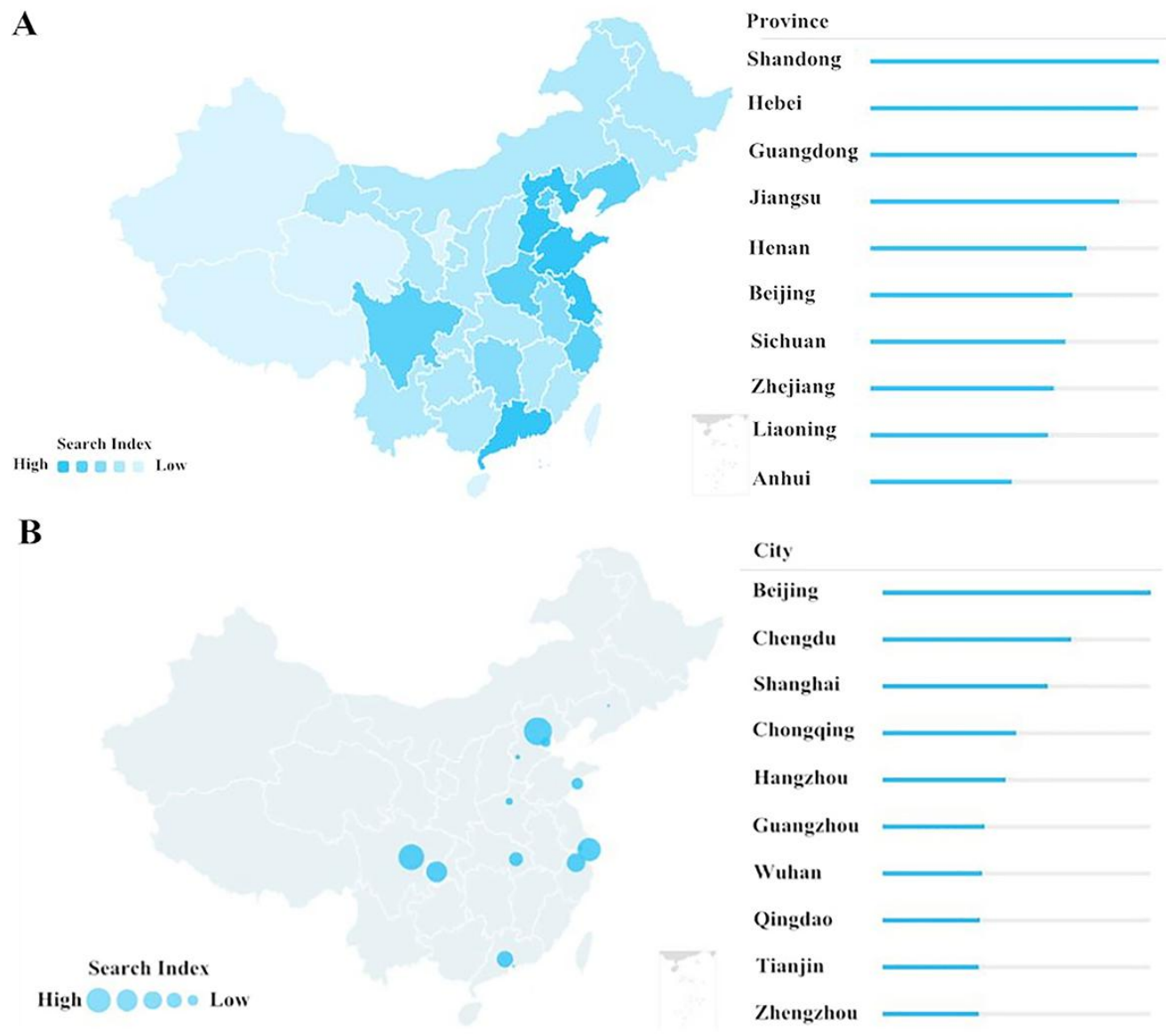

Figure 2. Geographic distribution maps for the population searching for information about "latest developments of epidemic" by province (A) and city (B), Jan 23, 2020-Feb 22, 2020. 
A

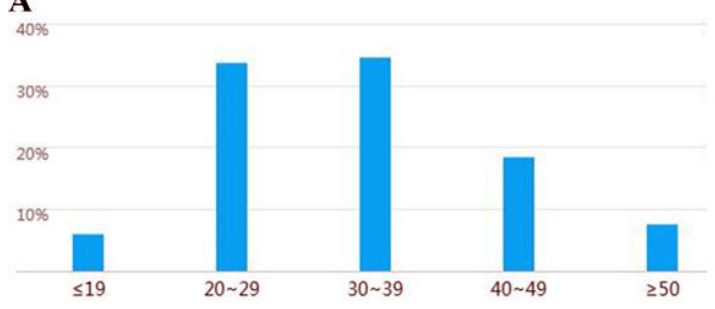

B

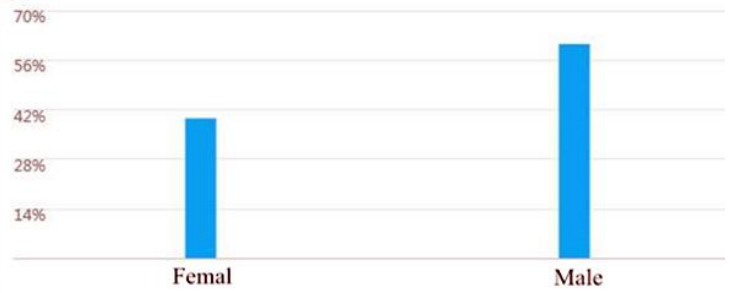

Figure 3 The demographic profiles by age (A) and sex (B).

A

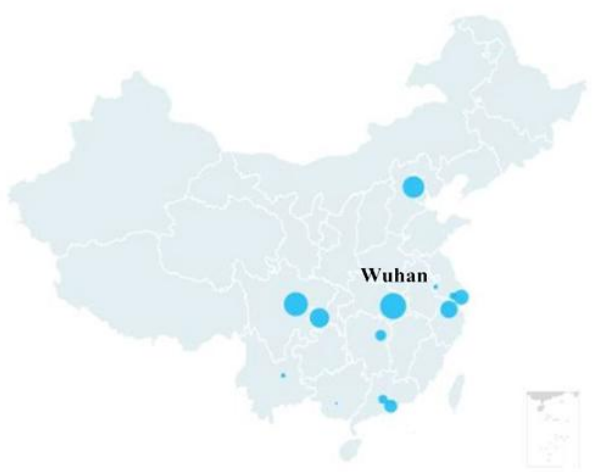

C

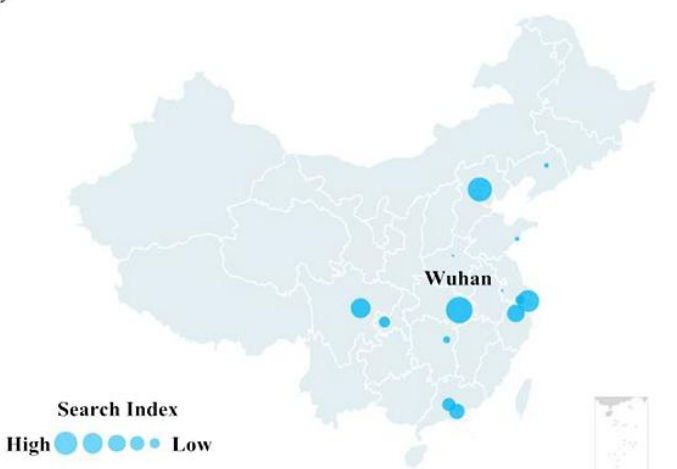

B

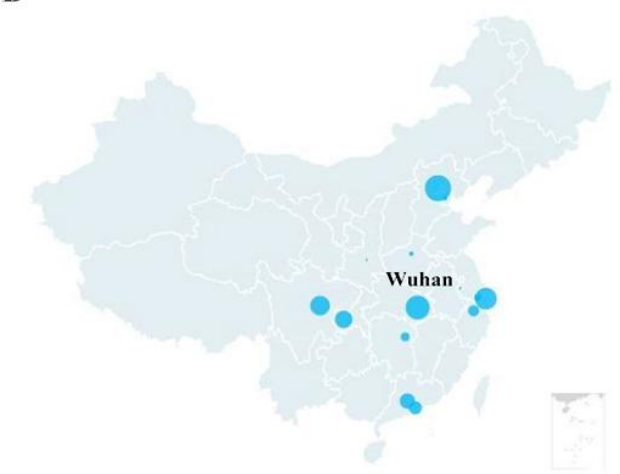

D

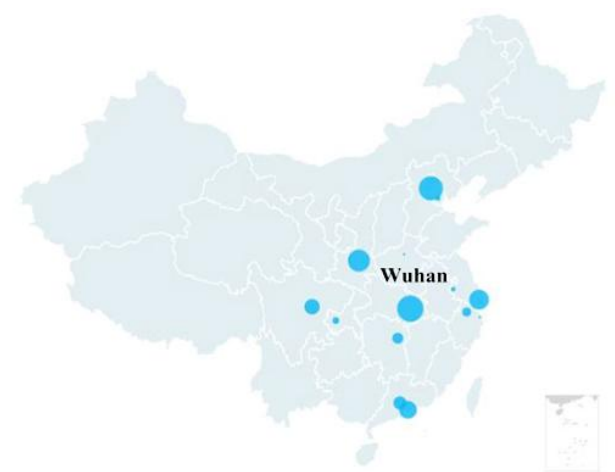

Figure 4 Geographic distribution maps showed that the most searched terms by Wuhan residents were "how to use a thermometer"(A), "n95 mask"(B), "testing kit" (C), and "nucleic acid testing"(D). 